\title{
Distal limb deficiencies-micrognathia syndrome
}

INSERM

\section{Source}

INSERM. (1999). Orphanet: an online rare disease and orphan drug data base. Distal limb deficiencies-micrognathia syndrome. ORPHA:1307

The distal limb deficiencies-micrognathia syndrome is characterized by the combination of symmetric severe distal limb reduction deficiencies affecting all four limbs (oligodactyly), microretrog nathia, and microstomia with or without cleft palate. 